\title{
Excellence in Research
}

\author{
Michael Arthur
}

\section{Introduction}

World-class universities are defined as providing an excellent undergraduate and postgraduate education, informed by both the quality and extent of their research activity and profile. They conduct research of the very highest international quality and their work informs some of the most important scientific, technical, arts, humanities and social developments in global human society.

Although such world-leading research features prominently, most world-class universities pride themselves in the quality of their graduates and their ability to contribute to global society. To be recognised as being among the very best universities in the world, both education and research must be at the very highest international level of quality, and there is evidence that both are having a significant impact on global society, through translation, innovation and policy formation.

The relentless pursuit of new knowledge is a characteristic of global human endeavour that is of fundamental importance to the future of our planet. Research excellence may be provided by universities, national institutes, government agencies or by industry, but it is critically important that it develops widely across the entire spectrum of global cultures. This diversity of engaged cultures is a critically important element of the most creative research environments and it is an essential element of solving the world's most complex problems.

\section{Definition of Research}

There are many different definitions of research. The OECD defines research as "creative work undertaken on a systematic basis in order to increase the stock of knowledge, including knowledge of man, culture and society, and the use of this stock of knowledge

\footnotetext{
M. Arthur $(\triangle)$

University College London, London, UK

e-mail: michael.arthur@ucl.ac.uk 
to devise new applications" [1]. This definition works well across the panoply of disciplines, particularly if the phrase 'new applications' is interpreted broadly to include learned contributions to the arts and humanities and to social and public policy.

\section{What Does Research Excellence Look Like}

Research excellence is clearly discipline specific, but a common characteristic of world-class researchers is that they are involved in shifting paradigms and creating new models of human understanding. They are actively involved in constructively criticising perceived wisdom in a specific field. Their views are usually based on new observations, including qualitative observations or quantitative scientific evidence, or new interpretations that ultimately support logical conclusions. Not infrequently, such observations are stimulated by the application of new technologies and methodological advances.

The world's greatest universities have a critical mass of individual researchers, or research teams, capable of performing at this level and a concentration of research excellence that allows or facilitates highly creative approaches to tackling some of the world's most complex and difficult problems across disciplines [2]. Such 'concentration' of research excellence is a fundamental concept that every institutional leader must foster and support. At the very highest levels of worldclass performance universities demonstrate this level of excellence, not only at the level of specific individuals, but also within and across multiple disciplines.

World-class research universities are typically engaged in trying to solve the most complex and intractable problems facing their own societies, or global society, in a coordinated and systemic manner across multiple departments, and in confident partnerships with other world-class institutions, local or global industry, governments and ministries and their health care, education, social care and public policy systems. Being an effective and seriously engaged partner is now an essential aspect of being a world-class university.

No world-class university can be universally excellent at everything and thus each institution must decide where to focus institutional efforts and resources. Institutional leaders must be bold enough to focus resources where they might have the greatest impact on research performance and profile. Deciding where an individual university can be truly distinctive and have the greatest international impact in its research activity is of fundamental importance to achieving world-class status.

\section{The Integration of Research and Teaching in World Leading Universities}

There are somewhere in the order of 17,000 higher education institutions across the globe and a conservative estimate suggests that at least 1,000 of these are actively and effectively engaged in research at scale. It should also be 
acknowledged that this research is equalled in volume by research conducted in non-university public institutions and by industry. This is an incredible force for the advancement of global human society and it is clear that high quality research is now widely distributed across the world.

Universities that are truly research-intensive must focus on ensuring that their students benefit from an education that is carefully and fully integrated with the research profile of the institution and its research partnerships. My personal philosophy is that all students (UG or PGT or PGR) should benefit from becoming involved in the process of 'research', irrespective of discipline. This is fundamentally important in developing students' life skills and equipping them for their future. By engaging in the research process, students will learn to become critical independent thinkers and problem solvers. They will understand how knowledge is created, how it inevitably shifts with time, and they will be exposed to, and become personally experienced in, dealing with uncertainties at the 'edge of knowledge'. They will also become more experienced in working in a team and actively engaged in improving their ability to communicate effectively. Graduates with this background experience are highly creative and of great value to the organisations that they join for employment. Many will become 'leaders of the future' because of their ability to research background information and analyse problems from first principles and subsequently exhibit confidence in employing innovative approaches and methods of problem solution.

\section{The Role of High Quality Doctoral Research Training}

There is a wide variety in the approach to doctoral level study across the globe. Most systems select the highest performing students at undergraduate and master's level study and then engage such individuals in developing their research skills through a combination of more detailed taught programmes on research methodologies and techniques as well as their own individual research projects. High quality and dedicated research supervision by experienced supervisors is essential and dual supervision (by primary and secondary supervisors, usually bringing different expertise) is now commonplace. In the UK, research funders, together with universities, have been involved in creating the concept of high quality doctoral training centres that provide world-class doctoral training and experienced supervision of the highest quality. Doctoral students must be exposed to training in ethics, research integrity and the importance of engaging the public in their research findings as well as being intensely focused in their own independent research projects. A critical mass of $\mathrm{PhD}$ students is essential to creating a sense of community and collegiality in a research-intensive university. This relates not only to the absolute number of PhD students in the university, but also to the manner in which they are organised. The concept of high quality experience as PhD students is a central feature of many graduate schools and doctoral training centres. At UCL we currently have in the order of $4900 \mathrm{PhD}$ students in our graduate school and doctoral training centres, in a total student population of just over 30,000 students. 
The duration of doctoral level training and research varies across the globe from 3 to 7 years and is also quite discipline specific. A typical such duration in the UK is four years. The centre piece of $\mathrm{PhD}$ student training is the creation of a doctoral thesis that is ultimately subject to detailed external scrutiny by the world's preeminent expert reviewers. The creation of new knowledge and an ability to demonstrate a logical and integrated set of arguments and research findings lies at the heart of every successful doctoral thesis.

\section{The Postdoctoral Period and Breaking Through as an Individual Researcher and Principal Investigator}

Successful completion of a $\mathrm{PhD}$ thesis is, however, only the beginning of a research career in most disciplines. A period of postdoctoral work and further study in the order of 3-9 years is now commonplace in most world-class universities, prior to individuals taking up their first academic appointment. This period usually involves moving to a different institution, or country, exposing postdoctoral students to a different research culture and, most importantly, to fresh ideas and techniques. In my opinion, this is the most formative period of any research career.

A world-class university will create a supportive and superb ongoing training environment for its postdoctoral staff, with continued expert oversight, support and advice, coupled with constructive challenge. During this period, postdoctoral staff will usually begin to develop and pursue their own independent ideas and acquire an increased level of expertise in their specific field. Wherever possible, highly talented postdoctoral staff should be encouraged to write grants for their own fellowship funding, such that they can formally, and more readily, branch out into an independent research career. Postdoctoral staff intending to pursue an academic career should also start to learn how to teach and ideally, they should gain experience and professional qualifications specific to teaching in a higher education institution during this period.

Inevitably, this period of development of the early stages of an academic career is highly variable around the globe. In some countries, it is possible to progress at an earlier stage to the junior phases of an academic career. There are also discipline specific variations. If however, you want to create a world-class research environment and profile for a university, then giving specific attention to this period of development of an independent research career is essential, as is the provision of ongoing mentorship, support and guidance. In the Western world, this is a phase of development through which only the very best successfully progress to develop their independent careers as principal investigators and this selectivity contributes to creating long-term world-class research performance in an institution. 


\section{The Importance of Cross-Disciplinary Research}

While a great deal of research of world-class quality will be confined to individual disciplines, there is an increasing recognition that many of the world's greatest problems need to be tackled by teams of researchers pulled together from different disciplines. At UCL we prefer the notion that each individual in a cross-disciplinary team brings their own disciplinary excellence to tackling a major problem and we therefore prefer the term 'cross-disciplinary' rather than 'inter-disciplinary' or 'trans-disciplinary'. These terms are often used interchangeably, but the latter two are perhaps more indicative of individuals from one discipline beginning to work in another disciplinary area. Such discipline hopping may be productive, but it is not always successful, and in our view it is important to bring disciplinary excellence to the table as research teams are formed. When tackling complex problems a cross-disciplinary approach is highly creative as individuals bring their discipline specific approaches to the table and share ideas and concepts across the team.

There are numerous perceived barriers to the creation of effective cross-disciplinary research teams and it is important that they are recognized and dealt with by institutional leaders. The most common problems relate to the discipline specific nature of financial and other organisational structures within our institutions. Moreover most external organisations, for example many research funders, journals and learned societies are organised by discipline and this creates the perception that it is easier to obtain funding, to publish and to achieve personal recognition and promotion by remaining safely within a discipline's confines.

It is important for institutional leaders to set a clear tone concerning the importance of cross-disciplinary research and to create opportunities for these activities to flourish. The rewards from getting this right are significant in terms of an institution's global research profile. How best to achieve this will vary from one institution to another. At UCL we have developed the concept of a 'grand challenge' approach, and have actively promoted these via a series of 'town meetings' with expert panels, informed debate and open discussion. This has been sufficient to initiate collaborative cross-disciplinary discussion that has led on to new research and educational activities.

Each grand challenge is guided forward by a senior academic executive team. Small pump-priming grants are available to help new projects get off the ground, but as new activities develop, external grants are sought. Our current grand challenges are in Global Health, Human Well-Being, Intercultural Interaction and Sustainable Cities [3]. This approach is now sufficiently embedded in our research culture that it is also informing our Global Engagement (internationalisation) strategy and our approach to massive open on-line courses (MOOCs) and online distance learning.

Other approaches to promoting cross-disciplinary activity in research and education can also be taken. In higher education environments where philanthropy is 
commonplace and generous, then it is not uncommon to see very large donations given specifically to promote such new 'centres'.

Another model is one that I learnt about from colleagues at Penn State University, USA, and then deployed at the University of Leeds, during my time there as Vice-Chancellor. This can be described as a 'co-funding model' and in my experience, it is highly successful. In essence monies are gathered into a central strategic fund on an annual basis (for example by applying a $1 \%$ strategic surcharge), such that there is a central pot of recurrent funds to support cross-disciplinary activities of the highest quality. The highest quality projects are selected competitively via a bottom-up process, led by teams of faculty members. Such projects are then co-funded 50:50 by the centre jointly with the relevant departments, schools or faculties involved. Milestones of performance are set at the beginning of the co-funding award and these must include a significant return on investment in terms of raising external funding, industrial interaction, etc. Each cross-disciplinary activity funded in this manner is reviewed on a quinquennial basis and if successful, funding is continued for a second five-year period. One striking example of great success using this co-funding model was the creation of a new cross-disciplinary group in Leeds, who were interested in Water and its management in flood and drought, called Water@Leeds [4]. This is now a highly successful and internationally renowned group, achieving a return on investment (research $£$ raised, compared to $£$ invested), of greater than 10:1.

\section{The Importance of Competitive Research Funding}

The principal routes for funding of university research may vary considerably from country to country. There may be direct government funding of university faculty, research staff, projects and programmes, or there may be competitive government grant funding systems, charitable and generous funding, endowment support or funding directly from industry. Faculty members may have some or all of their salary funded directly by government, but in other jurisdictions, particularly the USA, many faculty members raise some or all of their salary via grant funding. There are relatively few sources of funding for international collaborative funding, with the most important being the European Union framework funding (the current programme is called Horizon 2020). More recently, the European Research Council has become a very important international source of funds for high quality response mode funding.

In the UK there is a dual funding system for the support of research. Each university receives a direct government grant, the size of which depends on an intermittent assessment of research performance, which is undertaken every 6-7 years. This used to be called the Research Assessment Exercise (RAE), or as it is known in its most recent form, the Research Excellence Framework. A detailed description of this process is available at [5]. 
In brief, it involves each member of academic staff submitting their best four research outputs over the preceding period, together with a description of the research environment, research strategy and plan, and evidence of the impact of that research activity in the form of 'impact case studies'. For UCL this system currently informs the award of approximately $£ 115 \mathrm{M}$ per annum, as one limb of the dual support system.

The other element of dual support comes through government funding of the UK research councils, which is a typical peer-reviewed grant awarding system, largely organised by cognate groups of disciplines. This system drives the funding of individual research projects and programmes through a system of peer-reviewed grant awards, made to research teams or individual researchers via their institutions. From such government and other sources (charities, EU, Industry, other government departments and the UK National Institutes of Health Research) UCL achieved a total research income of $£ 375 \mathrm{M}$ last year.

Competition for research funding based on peer-reviewed grant submissions is commonplace across many countries, as it is held up as the best way of distributing scarce research funds (and in many cases, public money) to the highest quality research. In my experience, this mode of funding is a critically important element in driving forward personal research excellence. It is very demanding and exacting to write a grant that competes at the highest level, but in doing so, the quality of thought, preparation, methodology and preliminary data must all be in place, together with a carefully laid out research plan, prior to funding being ultimately awarded.

In many fields, this drives individual researchers to either acquire or develop the most advanced research methodologies that then allow them to tackle the more interesting and complex problems. This is particularly, but perhaps not exclusively, evident in Biomedicine and Biomedical Engineering in recent years, with the explosion of, for example, genetic manipulation techniques.

This competitive nature of research funding inexorably drives quality upwards across the entire research endeavour. It is clearly not a prerequisite for research excellence, but it certainly helps to drive it forward on a system-wide basis. In my opinion, the highly competitive nature of both limbs of our dual funding system is the key factor underlying the excellent, internationally competitive, research performance of the UK.

\section{Writing Successful Research Grants}

One of the most important transitions that every successful researcher has to undergo is to develop from being a good researcher at the bench, or in the library, into an independent principal investigator (PI). This is a difficult transition, but it is often the time when new ideas are born and exciting new paradigms develop. Critical to becoming a PI is the ability to attract competitive peer-reviewed grant funding, to allow you to explore your own ideas. 
Grants that are funded have several key elements.

- First and foremost, they must tackle an interesting problem or concept that excites the funding agencies and their boards and peer-reviewers to the extent that they envisage the results of the research having a significant impact on the field.

- Many will have substantial arguments and/or preliminary data that support the case being made for funding. In my view, time spent on acquiring additional preliminary data to strengthen the case is extremely valuable.

- They must have a clear and testable hypothesis and seek to deploy advanced technologies to help address some of the most complex of problems. For example, within the sciences, research that determines new mechanisms is of greater importance than that which is merely descriptive.

- They must be very clearly written, such that peers can quickly assimilate the key points and are thus guided through the grant in the direction of a supportive decision.

In many years of active research, I gave the utmost care and attention to my grant writing skills and fortunately enjoyed considerable success. I was never complacent and I always asked my close colleagues and fellow professors (at least two independent and knowledgeable scientists) to read and critique my grants, well in advance of their submission. If that meant that I had to miss a grant deadline and submit later, then I always accepted that outcome. There is nothing more important than ensuring that grants are of the very highest calibre at the time of submission.

\section{Research Leadership}

World-class universities will typically be home to a significant number of outstanding research leaders and they will support and develop others to take up research leadership roles. Great research leaders have a number of important characteristics. First and foremost they are prominent figures in their respective fields through their own proven research excellence, which has usually been sustained over a prolonged period of time. They will be publishing at the highest international level, giving plenary session keynote speeches at international meetings, and perhaps winning major awards such as the Nobel Prize, the Kavli Prize, the Lasker Award or the Fields Medal.

They typically have a great awareness of the history of and a good feel for the future of their discipline. They are influential, set the pace for others to follow and tend to shape the future direction of the field. This is typically associated with an ability to constantly evolve their own research strategy, a significant level of personal persistence in seeking answers to complex research questions and a willingness to take risks in their approach. 
Research leaders also support and nurture younger researchers and act as their mentors. The very best are generous with their time and create a research environment where others can flourish and follow in their footsteps. They are usually exceptionally talented, highly creative and effective at pulling together significant levels of research funding and they act as a centripetal force in terms of attracting the very best of the next generation (PhD students, postdoctoral staff and new faculty members) to come and work with them. They generate excitement and great research momentum in their home institution and act as role models for future generations of researchers.

Many of the skills required to become an excellent leader can be identified and taught through research leadership programmes that are aimed at up-skilling the younger generation of researchers. We have one such course at UCL, called 'Leadership in Action' [6].

This course is a three-day intensive programme designed to prepare researchers for leadership in their chosen research field or within the wider university community. The course is aimed at helping early career researchers to explore the concept of leadership and to develop confidence in their leadership style. It is intended to expose individuals to a variety of choices in how they lead and to provide them with understanding of the impact that may have on those they are leading. It is intended that course participants will learn how to influence people effectively towards a shared goal and that they will understand how all these skills will benefit them in their current research roles and beyond. The course is based on practical, experiential learning, rather than lectures, and it includes opportunities for all participants to lead a group project. Support and feedback are provided throughout the course on leadership style by expert coaches. It is our intention that this course will generate a cadre of early career researchers, equipped and skilled to become the distinguished research leaders of the future.

\section{Research Integrity, Handling Research Data and Research Misconduct}

It is incumbent on every world-class university to adhere to and promote the very highest standards of research integrity. This means creating an environment, which ensures that all research is conducted against a background of awareness of the key issues of transparency, honesty, collegiality, fairness and personal responsibility. Individual researchers (staff and students alike) must be trained in, and aware of, the institution's, research funding agency's and publisher's research policies and practices, and must adhere to them and to the highest possible standards of good research conduct in their daily activity. This must include excellence in the design of research projects and frameworks, their subsequent operation and adherence to the principle that it is the personal responsibility of all involved to ensure 
that research data is robust and reproducible. Researchers must therefore demonstrate intellectual honesty at all times.

The very highest standards of research integrity encompass many different elements, all of which require compliance. In addition to those outlined above, these include:

- Research ethics - all research should be conducted within the overarching framework of an ethical code. This is particularly important (and in most countries mandatory) if the research involves human subjects or animals.

- The requirement to declare any actual, potential or perceived conflict of interest, whether pecuniary or non-pecuniary.

- A responsibility to ask questions, to be aware and to report any reasonable suspicion of research or ethical misconduct.

- A responsibility to record and store original research data in a format that substantiates the reporting and publication of research findings and that is accessible to others, if required. Many funding agencies and publishers have specific requirements for the duration of data storage of original data and it is critically important that such requirements are strictly adhered to. Research data must be stored in a secure manner, such that it remains authentic and complete.

- Fairness and collegiality when dealing with research colleagues and collaborators, including the need to demonstrate fairness in peer review.

- A requirement to avoid misrepresentation of personal contribution to a specific research project or publication.

It is important that institutions also have systems to identify, investigate and deal with research misconduct when and if it occurs. The latter takes many forms and this has been outlined by Research Councils UK as follows [7]:

- Fabrication, or creation, of false data, or research documentation, such as consent to participate.

- Falsification, which comprises the inappropriate manipulation and/or selection of data, imagery or consent.

- Plagiarism, which comprises the misappropriation or use of others' ideas, intellectual property or work (written or otherwise) without acknowledgement or permission.

- Misrepresentation of data, duplication of publication, material interests, qualifications or experience, and of involvement in research.

- Breach of duty of care, whether deliberately, recklessly or by gross negligence to include breach of confidentiality, placing individual research subjects in harm's way, or not observing legal, ethical or statutory requirements and improper conduct in peer review, including failure to disclose conflicts of interest.

- Improper dealing with allegations of misconduct, including attempts at 'coverup' or reprisals against whistle-blowers, and failing to deal appropriately with malicious allegations. 
The websites [8-10] provide a more detailed background to this subject for those looking for more in-depth information and for further guidance on how to promote good research conduct or how to further handle research misconduct.

\section{From Research to Innovation and Enterprise}

World-class universities drive innovation, entrepreneurship and enterprise through the generation of intellectual property and new ideas. They contribute to supporting local, national and international businesses and entrepreneurs and they help to generate economic growth. This relates, of course, to the commercial exploitation of their research activities, but is also related to the supply of graduates as highly talented individuals that contribute to the ongoing development of an economy. There are many different ways in which universities can contribute to economic growth through their own enterprise activities. These include:

- The identification, protection and exploitation of intellectual property. All staff should be trained and supported by the University to recognise intellectual property and to learn how to protect it and where relevant, to exploit it. A dedicated professional team should be created to provide support for identifying novel concepts and ideas, which will not only develop strategies for their commercialisation, but also find investors to support them financially. The intention is that such a combined academic and professional endeavour, not only creates intellectual property, but also converts it into products of commercial value that can be licensed, and in some cases facilitated, to generate new spin-out companies. These, in turn, will create new products and businesses of long-term enduring commercial value. The complexity and risks involved in generating this level of success should not be underestimated, but the rewards for getting it right can also be significant. Guidance and advice should be sought from individuals that have successfully negotiated this space and that have first-hand experience of creating such high tech companies from their own intellectual property. Universities increasingly need to demonstrate to their own governments, and other funding agencies, that both their basic and applied research can bear such fruit.

- The recognition that world-class universities must also support their students and staff to be entrepreneurial at a personal level is an increasingly important paradigm. This should include the recognition that new student and staff entrepreneurs will need advice to support and develop their own independent commercial ideas and business practice with potential for leading to new and successful businesses. Where possible, universities should provide the facilities within which student and staff entrepreneurs can conduct research of the highest calibre and further develop their own ideas. The facilities and the expertise contained therein should be readily accessible to businesses external to the University so that research and development ideas can be positively exploited. 
- World-class universities also need dedicated individuals and systems to interact with the external business world to provide expert consultancy and to support corporate partnerships. These are specialist areas, and individuals or teams dedicated to this sphere of external partnerships are important in presenting a professional interface for interaction with the business and corporate world. Such teams can also be extraordinarily important in improving the profile and reputation of a university with the corporate world on a global scale. Dedicated teams in this area are also extremely helpful in guiding academic staff's navigation around this (perhaps previously) unfamiliar world and this helps enormously in managing risk and reputation.

\section{Five Key Actions for KAU to Enhance Research Performance}

King Abdulaziz University (KAU) is already a highly successful research-intensive university and a leading institution in the Middle East, with an established international profile. It is well led and has chosen to pull together a highly experienced, high profile International Advisory Board, of which I have the pleasure of being a member. I am still learning about KAU, and the following advice on five key actions is offered against that background.

(1) It is essential to build both a critical mass of researchers and a concentration of research excellence in a defined and clearly identified number of high quality fields of research. Choices must therefore be made and resources focused on ensuring that these 'peaks of excellence' can perform at the highest level. Human capital in the form of world-class academic and research staff is the most important element of building this level of success.

(2) As research performance improves, it is essential to think of the research pipeline and to recognise the importance of a clear 'youth policy' with respect to training the next generation. High quality doctoral training is essential, but this must also be aligned to very clear ongoing support and training during the postdoctoral period and into the early phases of a junior academic career.

(3) Seeking external research funding from high quality research funding agencies that use international quality peer review is an excellent way of sharpening research protocols and for enhancing research programmes. It also drives the need to stay abreast of methodological advances, which ultimately feed into promoting the international quality of research activity.

(4) Systems should be put in place to support and enhance cross-disciplinary research, allowing teams of researchers from many different disciplines to come together in order to tackle complex global and societal problems. Contributions to teamwork and working across disciplines must be recognised within the university's promotion and reward systems. 
(5) Research findings are at the forefront of the process of innovation, but research alone does not drive economic growth and prosperity. Systems must be put in place to drive and support innovation such that new products can be licensed and/or new companies spun out of the university. This should include the concept of expert teams to encourage and support entrepreneurship, consultancy and greater interaction with industry.

\section{Summary and Conclusions}

The advancement of human society is critically dependent on the creation of new knowledge through the conduct of research in universities, institutes, government agencies and industry. In our global future, the world's greatest problems will be tackled by teams of researchers from diverse cultures working across disciplinary, sectoral and national boundaries. World-class universities will be at the heart of this endeavour in many different ways. Our academic staff will not only be leading the immediate and long-term research effort, but they will also be closely involved in the training of the next generation of researchers through undergraduate, master's, and doctoral level education and research supervision. The close integration of education of students (at every level) with research activity will continue to define the world's leading universities.

Building a successful research-intensive university requires excellent institutional leadership that focuses on building world-leading quality in a defined number of research areas, as well as providing world-class research facilities. The ability to attract the very best research-active academic and postdoctoral staff and $\mathrm{PhD}$ students is of fundamental importance. Bidirectional student and staff mobility are of crucial importance for the creation of a positive research environment. Supporting these staff in their competitive grant writing is also critically important. Instruction on the key elements of ethics, research integrity and the manner in which primary research data should be handled and stored are now fundamentally important aspects of research training.

The future of the world's leading universities is very exciting as we continue our current journey along the road of the ever-increasing globalisation of higher education. Those universities that rise to the challenges outlined in this chapter will be best placed to make significant impact and thereby achieve world-class university status in recognition of their contribution to the advancement of knowledge and the future of humankind.

Open Access This chapter is distributed under the terms of the Creative Commons Attribution Noncommercial License, which permits any noncommercial use, distribution, and reproduction in any medium, provided the original author(s) and source are credited. 


\section{References}

1. OECD. (2002). Frascati manual: Proposed standard practice for surveys on research and experimental development (6th ed). Retrieved 27 May 2012 from www.oecd.org/sti/frascatimanual

2. Jewels in the Crown: The importance and characteristics of the UK's world-class universities. http://russellgroup.org/JewelsInTheCrown.pdf

3. http://www.ucl.ac.uk/grand-challenges

4. http://www.wateratleeds.org

5. http://www.ref.ac.uk

6. http://www.ucl.ac.uk/hr/od/pdp/sdlia/index.php

7. http://www.rcuk.ac.uk/Publications/researchers/grc

8. http://www.grad.ucl.ac.uk/research-integrity

9. http://www.admin.ox.ac.uk/researchsupport/integrity

10. http://www.universitiesuk.ac.uk/aboutus/AssociatedOrganisations/Partnerships/Pages/Resear chIntegrity.aspx 